\title{
CATS: CfAO Treasury Survey of distant galaxies, supernovae, and AGN's
}

\author{
David C. Koo ${ }^{1,4}$, Jason Melbourne ${ }^{1}$, Claire Max ${ }^{1,4}$, Anne Metevier ${ }^{1,4}$, \\ Mark Ammons ${ }^{1}$, James E. Larkin ${ }^{2}$, Matthew Barczys ${ }^{2}$ Shelley A. \\ Wright $^{2}$, and Eric Steinbring ${ }^{3}$ \\ ${ }^{1}$ Dept. of Astronomy and Astrophysics, University of California, Santa Cruz, CA 95064, USA \\ email: ammons@ucolick.org, anne@ucolick.org,jmel@ucolick.org, koo@ucolick.org, \\ max@ucolick.org \\ ${ }^{2}$ Dept. of Physics and Astronomy, University of California, Los Angeles, CA 90095, USA \\ email: barczysm@astro.ucla.edu, larkin@astro.ucla.edu, saw@astro.ucla.edu \\ ${ }^{3}$ Herzberg Institute of Astrophysics, NRC Canada, Victoria, BC V9E 2E7, Canada \\ email: Eric.Steinbring@nrc-cnrc.gc.ca \\ ${ }^{4}$ UCO/Lick Observatory, University of California, Santa Cruz, CA 95064, USA
}

\begin{abstract}
The NSF Science and Technology Center for Adaptive Optics (CfAO) is supporting a major scientific legacy project called the CfAO Treasury Survey (CATS). CATS is obtaining near-infrared AO data in deep HST survey fields, such as GEMS, GOODS-N, \& EGS. Besides summarizing the main objectives of CATS, we highlight some recent imaging work on the study of distant field galaxies, AGNs, and a redshift $z=1.32$ supernova. CATS plans the first data release to the community in early 2007 (check http://www.astro.ucla.edu/ irlab/cats/index.shtml for more details on CATS and latest updates).
\end{abstract}

Keywords. Instrument: adaptive optics; surveys; galaxies: active; galaxies: evolution; supernovae

\section{Why CATS?}

CATS is a long-term survey aimed for an enhanced near-IR study of the structural, chemical, star formation, and kinematic evolution of distant field galaxy subcomponents on sub-kpc scales. This is possible by using adaptive optics (AO) on an 8-10m class telescope that has diffraction-limits in the near-IR of 0.05 arcsec, which is $3 \mathrm{x}-4 \mathrm{x}$ better than is possible with HSTs $2.4 \mathrm{~m}$ mirror at a similar wavelength. This resolution corresponds to the sizes of bulges, disks, bars, spiral arms, and merger/interaction or lensing signatures at redshifts $z>0.5$ and improves the study of unresolved star forming knots, supernovae, and AGN's. It also matches the optical diffraction-limit of HST, so the CATS strategy is to work in well-studied HST survey fields with ACS images taken in 2 or more filters, including GOODS, GEMS, \& EGS. These regions are highly leveraged by deep Chandra, XMM-Newton, GALEX, and Spitzer data from space and optical, near-IR, submm, and radio data from the ground.

Keck's AO is presently limited to the near-IR, which complements the optical by providing superior penetration of dust-obscured regions and having higher sensitivity to old stars. For high redshift objects, the near-IR measures light that was emitted as restframe optical, including the important $H \alpha$ and [NII] lines for spectroscopy. Keck II now routinely provides laser guide star (LGS) AO (Wizinowich et al. 2006), which has opened new doors for many areas of astronomy (see Liu 2006 for an overview of the advantages, disadvantages, and potential of AO). With LGS AO supporting tip-tilt stars 
as faint as 18th mag within $\sim 60$ arcsec (van Dam et al. 2006) and good characterization of the off-axis point spread function (Steinbring et al. 2005), CATS now has, for the first time, access to $20 \%$ or more of the area of the special HST galaxy survey fields, and potentially over 1500 galaxies to be studied with AO. Prior to LGS, only about $1 \%$ was reachable on Keck since much brighter natural guide stars of 12 th mag were needed (Wizinowich et al. 2000). Thus much of the early distant galaxy research with Keck AO by Larkin et al. (2000) and Glassman, Larkin, \& Lafreniere (2002) were undertaken without having the benefit of adding HST data (c.f., Steinbring et al. 2004).

\section{Highlights}

To date, CATS has obtained AO data with Keck II for about 10 pointings or 5 square arcmin with the NIRC2 camera in GOODS, GEMS, and EGS. One Chandra X-ray galaxy in GOODS-S was found to have two red nuclei for which the 4 filter HST ACS photometry in the optical indicated either old stars or dusty, younger stars. The AO Kband photometry provided the critical discrimination and suggested that both were old. Thus we were witnessing a "dry merger" (Melbourne et al. 2005). In another study, M. Barczys (PhD 2006) exploited the higher sensitivity to stellar mass of near-IR photometry to discover that many of the apparent major mergers with roughly equal luminosities seen in the HST optical images are in fact minor mergers with unequal stellar masses. Finally, CATS has recently achieved an important milestone by measuring the very faint $(\sim 24$ mag Vega) $\mathrm{H}$ band flux (and thus the restframe $\mathrm{R}$ luminosity) of a high redshift ( $z=1.32$ ) supernova that was lying atop its much brighter host galaxy (Melbourne et al. 2006).

CATS plans to acquire more LGS AO images, mainly to study the host galaxies of AGNs and the colors of bulges and disks. With the advent of OSIRIS (Larkin et al. 2006), an AO compatible near-IR spectrograph with an integral field unit, CATS is poised to gather high spatial resolution 2-D spectroscopic data. A major goal of CATS is to provide the community with good quality AO data with which to explore its scientific potential. To this end, CATS intends to release some Keck AO data by early 2007.

\section{Acknowledgements}

We thank the Keck Observatory staff for years of help and for making LGS AO a reality. We thank S. Perlmutter and the Supernovae Cosmology Project team for providing CATS with the supernova. This work was supported in part by the NSF Science and Technology CfAO, managed by UC Santa Cruz under cooperative agreement No. AST-9876783. We close with thanks to the Hawaiian people for use of their sacred mountain.

\section{References}

Glassman, T. M., Larkin, J. E., \& Lafreniere, D. 2002, ApJ, 581, 865

Larkin, J. E., Glassman, T. M., Wizinowich, P., et al. 2000, PASP, 112, 1526

Larkin, J. E., Barczys, M., Krabbe, A., et al. 2006, SPIE, vol. 6269, 42

Liu, M. C. 2006, SPIE, vol. 6272, 14

Melbourne, J., Wright, S. A., Barczys, M., et al. 2005, ApJ, 625, L27

Melbourne, J., Koo, D. C., Max, C., et al. 2006, AJ, in press

Steinbring, E., Metevier, A. J., Norton, Stuart A., et al. 2004, ApJS, 155, 15

Steinbring, E., Faber, S. M., Macintosh, B. A., Gavel, D., \& Gates, E. L. 2005, PASP, 117, 847

van Dam, M. A., Bouchez, A. H., Le Mignant, D., et al. 2006, PASP, 188, 310

Wizinowich, P., Acton, D. S., Lai, O., et al. 2000, PASP, 112, 315

Wizinowich, P., Le Mignant, D., Bouchez, A. H., et al. 2006, PASP, 118, 297 


\section{Discussion}

Richard Mushotzky: Do you detect an AGN core in the Chandra sources?

DAVID Koo: We have not made a thorough study, so I do not know, but I do not recall seeing a blue point source inside a red spheroid component. 